\title{
COMPARAÇÃO ENTRE AS DISTRIBUIÇÕES NORMAL E DE WEIBULL PARA ANÁLISE DA RESISTÊNCIA À COMPRESSÃO DO CONCRETO
}

\section{Comparison between the normal and weibull distributions for analyzing the compressive strength of the concrete}

\author{
Jorge Michael Colman ${ }^{1}$, Paulo Eduardo Teodoro ${ }^{2}$, Matheus Piazzalunga Neivock ${ }^{3}$, \\ Gilson Secco Riva ${ }^{4}$, Sidiclei Formagini ${ }^{5}$
}

Recebido em 07 de março de 2014; recebido para revisão em 29 de abril de 2014; aceito em 18 de maio de 2014; disponivel on-line em 12 de novembro de 2014.

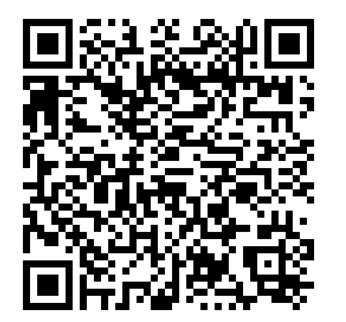

\section{PALAVRAS CHAVE:}

Estruturas de concreto;

Projeto estrutural;

Distribuição normal;

Distribuição de weibull.

\section{KEYWORDS::}

Concrete structures;

Structural design;

Normal distribuition;

Weibull distribuition.

\begin{abstract}
RESUMO: O projeto de estruturas de concreto possui uma modelagem matemática de natureza bastante subjetiva. Portanto, objetivou-se com esta pesquisa verificar se as distribuições Normal e de Weibull podem ser aplicadas aos dados resistências à compressão do concreto pronto, agrupados comercialmente. O estudo foi realizado durante o ano de 2011 na cidade de Campo Grande/MS. A resistência à compressão foi avaliada em ensaios de 189 amostras aos 28 dias a partir de diferentes construções de concreto armado realizados na cidade. Os ensaios ocorreram conforme prescrito pela NBR 5739 (ABNT, 2007). Para quantificar o grau em que a distribuição Normal e de Weibull se ajustaram os dados experimentais foram utilizados três testes de adequação: quiquadrado, Anderson-Darling e Kolmogorov-Smirnov. Com base no presente estudo, a distribuição Weibull pode ser aplicada aos dados de resistência à compressão para concreto. Isto sugere que, apesar de os complexos processos envolvidos na falha de compressão para um material compósito quase frágil como o concreto, um modelo de força estatística é eficaz. Além disso, ao comparar os testes de ajuste, há grande diferença prática entre as distribuições Normal e de Weibull. Esta informação é uma importante adição experimental para a literatura científica no que diz respeito à ruptura de materiais "semi-frágeis".
\end{abstract}

ABSTRACT: The design of concrete structures and their mathematical modeling is rather subjective in its nature. Therefore, it is the purpose of this study to see whether the Weibull or Normal distributions can be applied to the compressive strengths of commercially batched ready-mixed concrete. The study was conducted during the year 2011 in the city of Campo Grande / MS. The compressive strength was evaluated in 189 test samples at 28 days from different concrete constructions conducted in the city. The trials took place as prescribed by NBR 5739 (ABNT, 2007). To quantify the degree to which the proposed Weibull and Normal distributions fit the experimental data were utilized three goodness of fit tests: chi-squared, AndersonDarling, and Kolmogorov-Smirnov. Based on the present investigation, the Weibull distribution can be applied to compressive strength data for concrete. This suggests that, despite the complex processes involved in compression failure for a quasi-brittle composite material like concrete, a statistic strength model is possible. Furthermore, in comparing the goodness-of-fit, there is large practical difference between the Weibull and Normal distributions. The information is an important experimental addition to the body of knowl- edge regarding the failure of quasi-brittle materials.

\footnotetext{
* Contato com os autores: página seguinte
} 
* Contato com os autores:

${ }^{1}$ e-mail : Michael.colman14@hotmail.com (J. M. Colman)

Discente do curso de Engenharia Civil da Universidade Anhanguera-UNIDERP - Campo Grande / MS.

2e-mail : eduteodoro@uniderp.edu.br (P. E. Teodoro)

Discente do curso de Engenharia Civil da Universidade Anhanguera-UNIDERP - Campo Grande / MS.

${ }^{3}$ e-mail : matheus.neivock@ifms.edu.br ( M. P. Neivock )

Engenheiro de Materiais, Mestre em Engenharia de Materiais, docente do curso de técnico em Mecânica do Instituto Federal de Mato Grosso do Sul, Campus Campo Grande / MS.

${ }^{4}$ e-mail : mecfor@mecfor.com.br (G. S. Riva )

Eng. Civil, MECFOR - Tecnologia em Concreto Ltda. - Campo Grande / MS

${ }^{5}$ e-mail : formagini@yahoo.com.br ( S. Formagini )

Professor Dr. em estruturas do Centro de Ciências Exatas e tecnologia CCET da Universidade Federal de Mato Grosso do Sul - UFMS.

(C) 2014 REEC - Todos os direitos reservados.

\section{INTRODUÇÃO}

O projeto de estruturas de concreto possui uma modelagem matemática de natureza subjetiva. Para relacionar de forma direta ao custo dos dados de entrada, uma comparação mostra que analises determinista é mais barata, porem, seus resultados possuem uma validade limitada. As analises estatísticas fornecem ao projeto grande quantidade de informação, incluindo a distribuição das variações dos dados procurados. Entretanto, a aquisição dos dados de entrada é consideravelmente cara e, em alguns casos chega a custar tanto quanto o projeto ou o calculo da vida útil de estruturas únicas, seu uso é irrelevante devido à falta de conhecimento sobre os parâmetros de entrada (KASPÍSEK et al., 2010).

De acordo com os autores, à avaliação do nível de segurança das estruturas de concreto pode ser realizada considerando o comportamento estatístico dos principais parâmetros envolvidos. Particularmente em estruturas de concreto, a grande variedade de parâmetros mecânicos e reológicos pode dar origem a significantes desvios em relação ao comportamento esperado se uma abordagem determinista for usada. Por outro lado, já se sabe que a função de densidade da probabilidade e os parâmetros não podem ser univocamente definidos.

Heterogeneidade do concreto de cimento Portland invariavelmente causa diferentes resultados a partir de um dado concreto. Isto significa que um teste estatístico de forças é necessário (GIBB e HARRISON, 2010). Isso é feito geralmente assumindo uma distribuição normal e os parâmetros para descrever esta curva, ou seja, a força média "população" o desvio padrão é obtido a partir de um grande numero de resultados de ensaios (Rajaname et al., 2012). A Resistencia do concreto é considerada matematicamente como uma "variável aleatória" para o gráfico da curva de distribuição normal e marcada como abcissa (eixo x) (TUMIDAJSKI et al., 2006).

A configuração da distribuição normal da resistência do concreto é puramente fenomenológica. Não há base teórica para a escolha da distribuição normal. Há uma serie de outras distribuições que tem base teórica e pode dar uma melhor configuração aos dados. Uma delas é a distribuição de Weibull que é baseada no dado estatístico mais fraco para materiais frágeis, os mais resistentes são algumas vezes modelados por essa distribuição (WEIBULL, 1951).

À aplicação direta desse modelo na resistência a compressão do concreto é complicado por uma serie de fatores. Em primeiro lugar o concreto não é um material com comportamento frágil. O concreto é um material semi-frágil, o que significa que existe uma redistribuição dos esforços e de energia antes da ruptura (Bazant et al. 1991; Bazant and Novak 2000 a, 2000 b). Além disso, a ruptura do concreto devido as forças de compressão são causadas por varias pequenas fissuras que diminuem de extensão lentamente gerando uma zona esmagada (Yip et al. 1995; Carpinteri et al. 1999) ao invés da propagação rápida e estável das micro-fissuras, que é o mecanismo esperado na teoria do elo mais fraco 
(TUMIDAJSKI et al., 2006).

Portanto, o objetivo deste estudo é observar se o Weibull ou a Distribuição normal podem ser aplicados aos resultados dos ensaios de compressão do concreto comercial de mistura pronta. Essa informação é uma importante adição ao corpo de conhecimento sobre a ruptura de material semi-frágil.

\section{MATERIAIS E METODOS}

O estudo foi conduzido durante o ano de 2011 na cidade de Campo Grande / MS, os corpos de prova foram moldados in loco em várias obras. A resistência a compressão foi avaliada em 189 amostras após 28 dias da moldagem de diferentes construções executadas na cidade.

Os dados usados neste artigo foram fornecidos pelo laboratório de tecnologia em concreto MECFOR - Tecnologia em concreto Ltda. O concreto avaliado foi preparado em seis indústrias concreteiras instaladas no município de campo grande, MS. Os corpos de prova foram coletados de acordo com os procedimentos da NBR NM 33 (ABNT, 1998), e foram moldados de acordo com a NBR 5738 (ABNT, 2003) em obras de grande porte que realizaram controle de qualidade dentro do tempo especificado pela NBR 12655 (ABNT, 2006) e ensaiados em laboratório de acordo com a NBR 5739 (ABNT, 2007).

Os dados foram analisados por meio de planilhas do software Microsoft Excel, onde foram calculadas a média, o desvio padrão e o coeficiente de variação apresentadas na Tabela 1.

Para a distribuição normal da resistência do concreto aos 8 dias ( $\sigma$ ), a função de densidade da probabilidade, $f(\sigma)$, e a função acumulativa de distribuição, $F(\sigma)$, são, respectivamente, fornecidos pelas Equações 1 e 2 .

A função de densidade da probabilidade, $f(\sigma)$, e a função acumulativa de distribuição, $F(\sigma)$, para uma distribuição de Weibull de $\sigma$, são dadas, respectivamente, pelas Equações 3 e 4.

TABELA 1: Valores médios, Desvio padrão e coeficientes de variação da resistência a compressão em 189 corpos de prova aos 28 dias de diferentes construções na cidade de Campo Grande / MS.

\begin{tabular}{cc} 
Parâmetro & Valor \\
\hline Máximo & 43,70 \\
Mínimo & 26,95 \\
Média & 35,13 \\
Desvio Padrão & 3,65 \\
Coeficiente de variação (\%) & 10,38
\end{tabular}

$$
\begin{array}{cc}
f(\sigma)=\frac{1}{\sqrt{2 \pi . S}} \exp \left(-0.5 \frac{\sigma-\mu}{S}\right)^{2} & \text { Eq. [1] } \\
F(\sigma)=\phi\left(\frac{\sigma-\mu}{S}\right) & \text { Eq. [2] }
\end{array}
$$

Onde:

$\boldsymbol{\mu}$ : é a media da resistência à compressão aos 28 dias dos corpos de prova (MPa);

S: é o desvio padrão da resistência à compressão aos 28 dias dos corpos de prova (MPa);

$\phi$ : é a função de erro.

$$
\begin{gathered}
f(\sigma)=m\left(\frac{\sigma}{\sigma_{0}^{m}}\right)^{(m-1)} \exp \left[-\left(\frac{\sigma}{\sigma_{0}^{m}}\right)^{m}\right] \\
F(\sigma)=\phi\left(\frac{\sigma-\mu}{S}\right)
\end{gathered}
$$

Onde:

$\boldsymbol{\mu}$ : é a media da resistência à compressão aos 28 dias dos corpos de prova (MPa);

S: é o desvio padrão da resistência à compressão aos 28 dias dos corpos de prova (MPa); $\phi$ : é a função de erro. 
Para quantificar o grau em que o Weibull proposto e a distribuições normais ajustaram os dados experimentais utilizando três testes de adequação: chi-squared, Anderson-Darling, e Kolmogorov-Smirnov. O teste do qui-quadrado é um teste de área com base na função de densidade de probabilidade, e o Anderson-Darling e Kolmogorov-Smirnov são testes de distancia com base na função de distribuição cumulativa. Estes testes identificam exatamente qual a melhor distribuição para ajustar os dados avaliados.

\section{RESULTADOS E DISCUÇÃO}

$\mathrm{Na}$ tabela 2 foram delineados os resultados dos testes de adequação, onde todos revelaram uma melhor compatibilidade com a distribuição de Weibull nos resultados de Resistencia a compressão do concreto. Independente do teste, um valor menor de adequabilidade no ajuste dos testes estatísticos significa há um aumento na confiabilidade nos dados produzidos pela distribuição proposta.

Do ponto de vista estatístico, o chi-squared é um teste de distribuição de probabilidade. A confiabilidade deste tipo de teste é diretamente proporcional ao numero de experimentos, já os testes Anderson-Darling e Kolmogorov-Smirnov são testes de distribuição cumulativa que podem lidar de forma confiável com amostras de tamanhos menores. Portanto, na analise dos resultados da tabela 2 , há um pouco mais de confiabilidade no teste qui-quadrado. Consequentemente, A Distribuição de Weibull em geral é uma representação melhor do que a distribuição normal nos dados de resistência à compressão de concreto (Figuras 1 e 2).

\begin{tabular}{ccc} 
& \multicolumn{3}{c}{ TABELA 2: Testes de adequação de Weibull e distribuições normais. } \\
\hline & Distribuição Weibull & Distribuição Normal \\
\hline qui-quadrado & 29,50 a & 37,89 \\
Anderson-Darling & $0,68^{\mathrm{a}}$ & 1,49 \\
Kolmogorov-Smirnov & $0,04 \underline{\mathrm{a}}$ & 0,07 \\
\hline
\end{tabular}

ạ: Adequabilidade do teste.

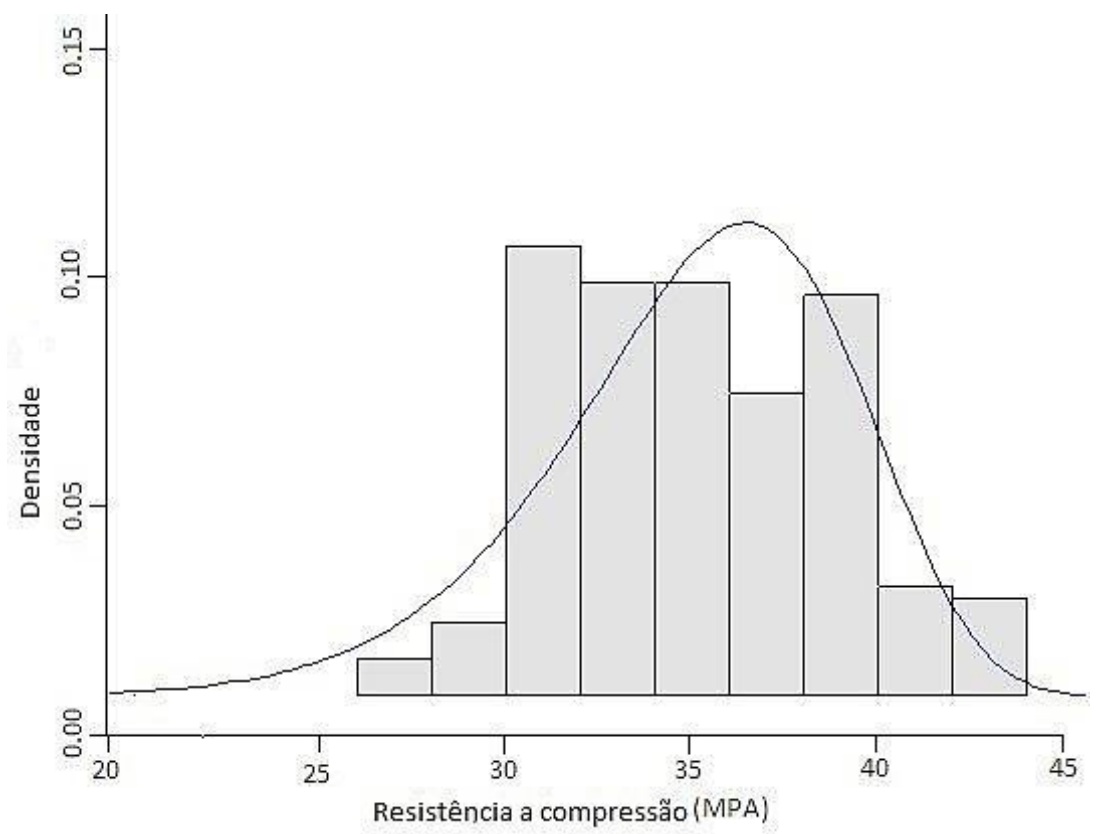

FIGURA 1: Distribuição Weibull para 189 valores de resistência a compressão de concreto. 


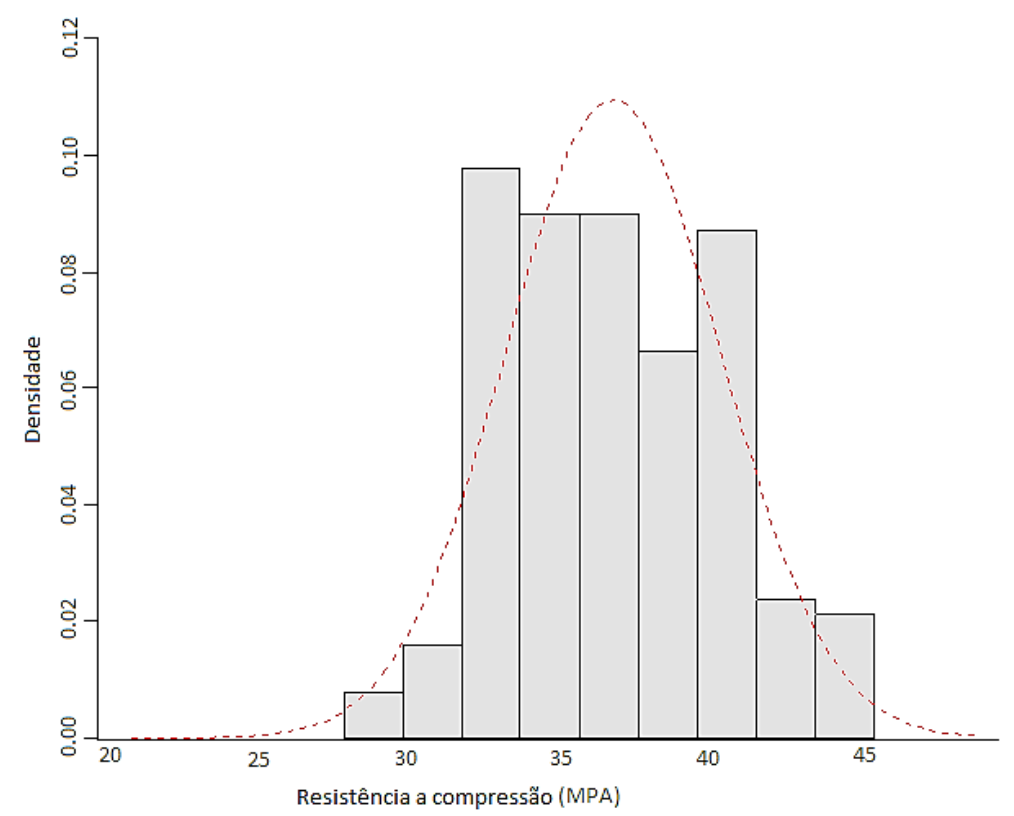

FIGURA 2: Distribuição Normal para 189 valores de resistência a compressão de concreto.

De acordo com Tumidajski et al. (2006) quando fizeram a comparação da distribuições para descobrir qual representava melhor o comportamento da resistência a compressão dos lotes de concreto comercial no Canada, não foi identificado diferenças significativas entre as distribuições a partir do resultado dessa pesquisa. Elgueta et al. (2007) para analisar qual a distribuição (Normal ou Weibull) é o mais adequado para os parâmetros de projeto de concepção estrutural, concluiu que a distribuição de Weibull é mais confiável, assemelhando-se a estes resultados.
As constantes " $\sigma_{0}$ " e " $m$ " são conhecidos como parâmetros de Weibull e esses parâmetros determinam a distribuição de tensão de ruptura. A equação 3 pode ser resolvida, e o resultado é uma linha reta com $\ln [-\ln (1-F)]$ na ordenada e In $\sigma$, na abcissa.

A distribuição de Wiebull permitiu encontrar a tensão de ruptura das amostras, em outras palavras $63.20 \%$ dos corpos de prova se romperam a uma tensão de $36.71 \mathrm{MPa}$, enquanto o parâmetro $\mathrm{m}=11: 51$ indica homogeneidade dos valores de resistência a compressão (Figura 3).

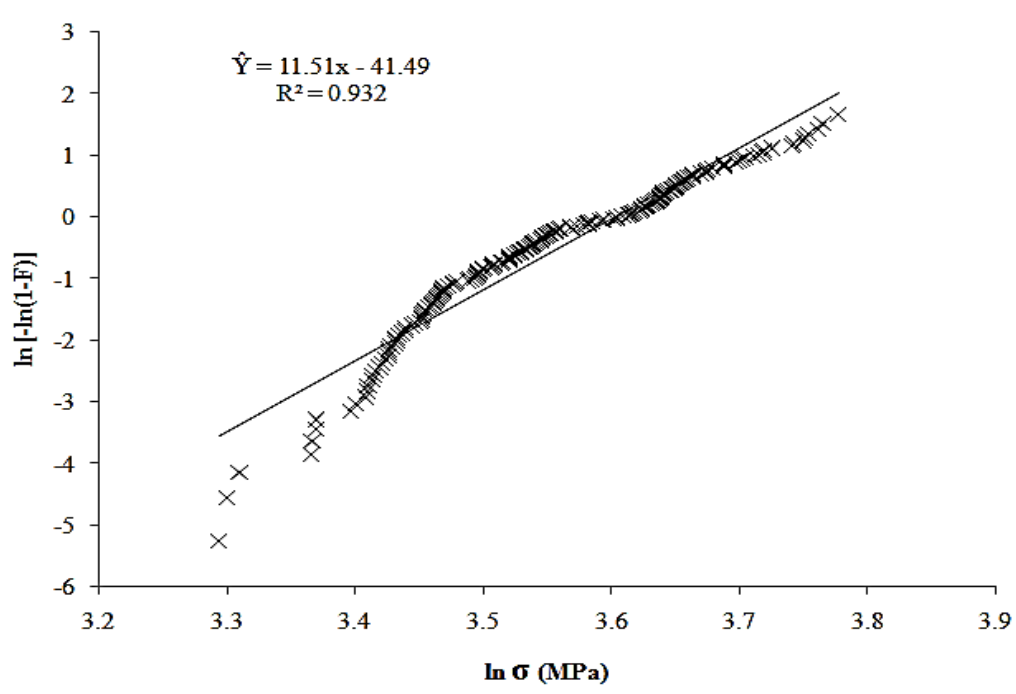

FIGURA 3: Gráfico Weibull para os dados de Resistencia a compressão. 


\section{CONCLUSÃO}

Baseado na presente investigação, a Distribuição de Weibull pode ser aplicada nos dados de resistência a compressão do concreto. Isso sugere que, apesar dos complexos processos envolvendo a ruptura a compressão de um composto semi-fragil como o concreto, a utilização de um modelo de força estatística é possível. Além disso, ao comparar a adequabilidade, há grande diferença pratica entre Weibull e a Distribuição normal.

\section{AGRADECIMENTO}

A empresa Mecfor Tecnologia em concreto Ltda. pela cooperação e condução dos ensaios.

\section{REVISÃO BIBLIOGRAFICA}

ASSOCIAÇÃO BRASILEIRA DE NORMAS TÉCNICAS. NBR 5738 - Concreto - Procedimento para moldagem e cura de corpos-de-prova. Rio de Janeiro, 2003.

ASSOCIAÇÃO BRASILEIRA DE NORMAS TÉCNICAS. NBR 5739: Concreto - Ensaio de compressão de corpos-deprova cilíndricos. Rio de Janeiro, 2007.

ASSOCIAÇÃO BRASILEIRA DE NORMAS TÉCNICAS. NBR 12655 - Concreto - Preparo, controle e recebimento. Rio de Janeiro, 2006.

ASSOCIAÇÃO BRASILEIRA DE NORMAS TÉCNICAS. NBR NM 33 - Concreto - Amostragem de concreto fresco. Rio de janeiro, 1998.

BAZANT, Z. P.; XI, Y.; REID, S. G. Statistical size effect in quasi-brittle structures. I. Is Weibull theory applicable? Journal of Engineering Mechanics, v. 117, n. 11, p. 26092622, 1991.

BAZANT, Z.P.; NOVAK, D. Energetic-statistical size effect in quasibrittle failure at crack initiation. $\mathrm{ACl}$ Materials Journal, v. 97, n. 3, p 381-392, 2000.

BAZANT, Z. P.; NOVAK, D. Probabilistic nonlocal theory for quasibrittle fracture initiation and size effect. II: application. Journal of Engineering Mechanics, v. 126, n. 2, p. 175-185, 2000.

CARPINTERI, A.; FERRO, G.; MONETTO, I. Scale effects in uni-axially compressed concrete specimens. Magazine of Concrete Research, n. 51, v. 3, p. 217-225, 1999.
ELGUETA, M.; DÍAZ, G.; ZAMORANO, S.; KITTL, P. On the use of the Weibull and the normal cumulative probability models in structural design. Materials \& Design, v. 28, n. 1, p. 2496-2499, 2007.

GIBB, I.; HARRISON, T. Use of control charts in the production of concrete. ERMCO, 2010. 53 p.

KARPÍSEK, Z.; STEPÁNEK, P.; JURÁK, P. weibull fuzzy probability distribution for reliability of concrete structures. Engineering MECHANICS, v. 17, n. 5/6, p. 363-372, 2010.

RAJANAME, N. P.; NATARAJA, M. C.; GANESA, T. P. A technical look at 'Individual test result' criterion for concrete acceptance as per IS 456:2000. The Indian Concrete Journal, v. 4, n. 1, p. 26-37, 2012.

TUMIDAJSKI, P.J.; FIORE, L.; . KHODABOCUS, T; LACHEMI, M.; PARI, R. Comparison of Weibull and normal distributions for concrete compressive strengths. Canadian Journal of Civil Engineer. v. 33, n. 1, p. 12871292, 2006.

WEIBULL, W. A statistical distribution function of wide applicability. Journal of Applied Mechanics, v. 18, n.1, p. 293-297, 1951.

YIP W. K.; TAM, C. T.; GARY, G. Concrete strength evaluation through the use of small diameter cores. Magazine of Concrete Research, v. 40, n. 143, p. 99-105, 1995. 